\title{
Advancing GHSA: Lessons learned about strengthening HIS and disease surveillance
}

\author{
Pia D. MacDonald ${ }^{\star}$, Niamh Darcy, Rita Sembajwe, Eileen Reynolds, \\ Henry Chidawanyika, Christopher Kelley, Michael McKay, Adam Preston and \\ Gordon Cressman
}

RTI International, Berkeley, CA, USA

\section{Objective}

The objective is to discuss two decades of international experience in health information and disease surveillance systems strengthening and synthesize lessons learned as applicable to implementation of the Global Health Security Agenda (GHSA).

\section{Introduction}

RTI International has worked on enhancing health information and disease surveillance systems in many countries, including The Democratic Republic of the Congo (DRC), Guinea, Indonesia, Kenya, Nepal, Philippines, Tanzania, Zambia, and Zimbabwe. Strengthening these systems is critical for all three of the Prevent, Detect and Respond domains within the Global Health Security Agenda.

We have deep experience in this area, ranging from implementing District Health Information Software (DHIS), electronic medical records, health facility registries, eHealth national strategies, electronic Integrated Disease Surveillance and Response system (eIDSR), mobile real-time malaria surveillance and response, national weekly disease surveillance, patient referral system, and community based surveillance. These experiences and lessons learned can inform work being done to advance the GHSA.

We will discuss several examples, including activities in Zimbabwe and Tanzania. RTI has been working in Zimbabwe for over six years to strengthen the national health information system. This work has included the configuration and roll-out of DHIS 2, the national electronic health information system. In doing so, RTI examined and revitalized the weekly disease surveillance system, improving disease reporting timeliness and completeness from $40 \%$ to $90 \%$. Additionally, RTI has integrated mobile technology to help more rapidly communicate laboratory test results, a laboratory information management systems to manage and guide test sample processing, and various other patient level systems in support of health service delivery at the local level. This work has involved capacity building within the ministry of health to allow for sustainable support of health information systems practices and technology and improvements to data dissemination and use practices.

Similarly, RTI has worked for more than five years to help strengthening the National HIS in Tanzania. These activities have included stakeholder coordination, developing national eHealth strategy and enterprise architecture, harmonizing indicators, redesigning routine reporting instruments, national DHIS 2 roll-out, information technology infrastructure management and user help desk support, reducing the number of parallel information systems, data dissemination and use, development of district health profiles, development of the national health facility registry, and supporting roll-out of the electronic integrated disease surveillance system.

\section{Methods}

We will profile selected projects and synthesize critical lessons learned that pertain to implementation of the GHSA in resource constrained countries.

\section{Results}

We will summarize our experience and lessons learned with health information and disease surveillance systems strengthening. Topics such as those that relate to advancing the GHSA Real Time Surveillance and Reporting Action Package areas will be discussed, including: indicator and event based surveillance systems; interoperable, interconnected, electronic real-time reporting system; analysis of surveillance data; syndromic surveillance systems; systems for efficient reporting to WHO, FAO and OIE; and reporting network and protocols in country.

\section{Conclusions}

Our experience working over the past 14 years in 9 countries on different HIS and disease surveillance system strengthening projects has led to a deep understanding of the challenges around implementation of these systems in limited resource settings. These experiences and lessons learned can inform initiatives and programs to advance the GHSA.

\section{Keywords}

Global health security; Surveillance; capacity building; Informatics

\section{Acknowledgments}

RTI acknowledges the support of the US Centers for Disease Control and Prevention, President's Emergency Plan for AIDS Relief (PEPFAR), US Agency for International Development, Bill \&Melinda Gates Foundation, and Qualcomm Inc. Additionally, RTI acknowledges the vast support and contributions of the many national and sub-national public health institutions and staff with whom we have collaborated.

\section{*Pia D. MacDonald}

E-mail: pmacdonald@rti.org 